\title{
aspects du comportement des sols non saturés
}

\section{some aspects of the behaviour of non-saturated soils}

\author{
P. DELAGE \\ Maître de Conférences à l'École Nationale \\ des Ponts et Chaussées \\ Chercheur au C.E.R.M.E.S. ${ }^{*}$
}

\section{Résumé}

A partir des travaux publiés dans le domaine, on présente les concepts de base nécessaires à la compréhension des problèmes posés par le comportement des sols non saturés, concernant le développement des pressions négatives, et les aspects hydrauliques et mécaniques. On examine la validité du concept de contrainte effective étendu au cas des sols non saturés, et les approches existantes des phénomènes de compression, consolidation et résistance au cisaillement. La complexité du problème, et la faible quantité de résultats expérimentaux existant rendent nécessaire le prolongement des recherches sur ce thème. On peut espérer ainsi arriver à mieux comprendre les troubles affectant les massifs compactés de certains ouvrages en terre soumis à des conditions météorologiques ou hydrauliques variables (remblais, barrages), et à proposer des méthodes de dimensionnement prenant en compte la non-saturation.

\section{Abstract}

Some basic concepts necessary to understand the behaviour of non-saturated soils and related to previously published studies are presented. These concern the phenomenon of suction, and hydraulic and mechanical problems. The concept of effective stress applied to non-saturated soils is examined, compression, consolidation and shearing strength problems are described. It is concluded that the problem is complex, and that the small amount of available experimental data need to be completed. It will thus be possible to provide a better understanding of some troubles which affect earth structures submitted to weather changes, and to define more adapted design methods.

\footnotetext{
- E.N.P.C. - C.E.R.M.E.S., B.P. 105, 93194 Noisy le Grand Cedex.
} 
Les développements de la Mécanique des Sols ont jusqu'à présent principalement concerné les sols saturés. puisque les travaux en la matière ont surtout été menés dans les pays tempérés. Cependant, la nécessité de prendre en considération le comportement des sols non saturés se fait actuellement particulièrement ressentir. Initialement, cette nécessité a été mise en évidence dans les pays à climat sec ou aride, où les phénomènes d'évaporation et de précipitations font varier de façon importante les profils de teneur en eau de la surface des massifs de sols, sur une profondeur caractéristique qu'on appelle la zone active. Suivant la nature du sol, et dans le cas de sols fins, ces variations peuvent entraîner des désordres dus à des phénomènes tels que le gonflement des argiles, ou l'effondrement des limons. Dans les climats tempérés, la mise en place de sols compactés fait également intervenir des phénomènes de non-saturation, où les problèmes d'infiltration, de remontée capillaire, et d'évaporation sont importants, et peuvent se traduire par des gonflements ou des fissurations. C'est le cas par exemple des remblais compactés.

Il est donc nécessaire de parvenir à une meilleure compréhension des phénomènes de non-saturation, dont la caractéristique principale est le développement, au sein de la structure du sol, de ménisques capillaires entraînant une valeur négative de la pression de l'eau. encore appelée succion.

\section{SUCCION}

\section{Phénomènes physiques}

Comme le montre la figure la, dans le cas des sols grenus, la localisation des ménisques eau-air se fait en affectant, au fur et à mesure du séchage du sol, des pores de dimensions de plus en plus faibles, se situant au contact entre grains.

La relation entre le rayon de courbure du ménisque et la différence de pression entre les phases gazeuse et aqueuse est définie par la loi de Laplace, qui se simplifie dans l'hypothèse de pores cylindriques et prend l'expression de la loi de Jurin :

$$
\mathrm{u}_{\mathrm{a}}-\mathrm{u}_{\mathrm{w}}=\frac{2 \tau \cos \theta}{x}
$$

où $u_{b}$ est la pression d'air, $u_{w}$ la pression d'eau, $\pi$ la tension de surface eau-air, et $\theta$ l'angle de raccordement ménisque-solide.

Par convention, on prend pour nulle la valeur de la pression atmosphérique. La pression d'eau devient donc négative, et la succion $u_{a}-u_{w}$ est d'autant plus forte que le rayon de courbure est faible.

Une autre relation issue de la thermodynamique existe entre la tension de vapeur d'eau de la phase gazeuse $\mathrm{P}$ au voisinage du ménisque eau-air et la succion, donnée par la loi de Kelvin :

$$
u_{a}-u_{w}=\frac{R T}{g M} \ln \frac{P}{P o}
$$

où Po est la tension de vapeur de l'eau pure libre, $\mathrm{M}$ la masse molaire de l'eau. $\mathrm{g}$ l'accélération de la pesanteur, $\mathrm{R}$ la constante des gaz parfaits et $\mathrm{T}$ la température absolue.

On note que cette relation est fonction de la température, et que l'humidité relative de l'air à proximité du ménisque est inférieure à la pression de vapeur saturante, et d'autant plus faible que le rayon de courbure est faible.

Dans le cas des sols fins non saturés, l'interaction eausolide n'est pas seulement due à la capillarité, mais également à l'adsorption de leau sur les particules solides. Parmi les phénomènes engendrant cette adsorption, on peut citer (MITCHELL, 1976) : la liaison hydrogène entre les molécules d'eau et les atomes d'oxygène ou d'hydroxyde situés sur la surface du feuillet argileux; l'eau nécessaire à l'hydratation des cations attirés électriquement par les défauts de charge du feuillet: l'eau attirée par l'attraction osmotique créée par la concentration de cations au voisinage du feuillet: l'attraction électrique du pôle positif du dipôle que forme la molécule d'eau: les forces d'attraction de Van der Walls.

Ainsi, alors que l'eau des sols grenus non saturés est soumise à un potentiel d'attraction capillaire, l'eau des sols fins non saturés est soumise à la somme d'un potentiel capillaire et d'adsorption, qu'on appelle globalement potentiel matriciel.

Il peut exister également, dans le cas de concentration en sels de l'eau interstitielle variables, un potentiel de nature osmotique. Il semble cependant que l'influence

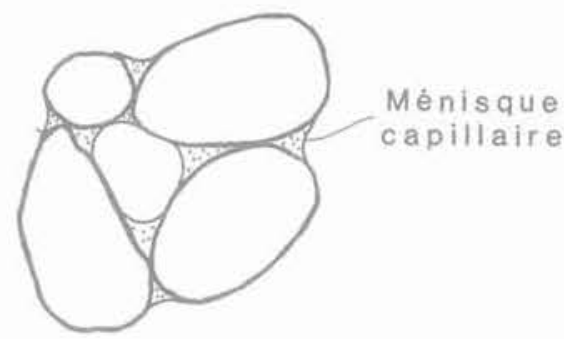

Sol granulaire

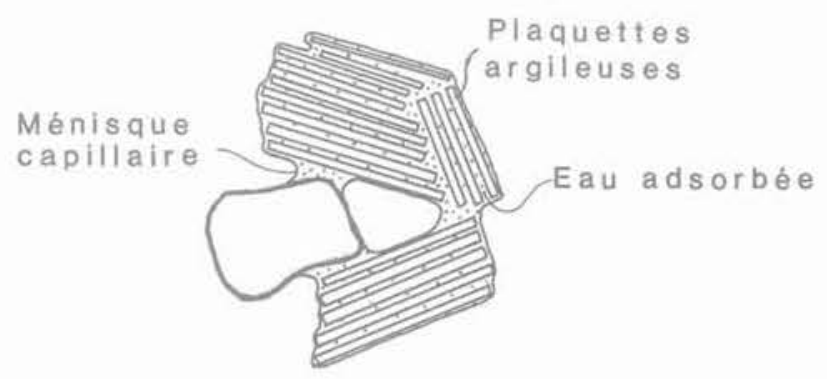

Sol fin

Fig. 1. - Phénomènes engendrant la succion. Fig. 1. - Suction phenomenon. 
de ce potentiel soit plus importante dans le domaine de la pédologie, et assez négligeable en géotechnique. Enfin, il convient de rappeler également l'existence classique des potentiels de pression et gravitaire,

\section{Courbes de rétention d'eau, $\mathrm{pF}$}

Pour un sol donné, des variations du degré de saturation vont entraîner des variations des valeurs de succion, qui seront différentes suivant le type de sol. Il est important, pour chaque sol, de connaître ces variations de la succion en fonction de la teneur en eau, qui correspondent à la courbe de rétention d'eau, également appelée courbes de succion, ou de pF.

\section{Détermination expérimentale}

La façon la plus couramment utilisée pour la détermination expérimentale des courbes de rétention d'eau consiste à imposer une succion $\mathrm{u}_{6}-\mathrm{u}_{\mathrm{w}}$ connue à un échantillon, en lui appliquant une pression d'air $\mathrm{u}_{\mathrm{s}}$ positive, et en gardant la pression d'eau égale à la pression atmosphérique $\left(u_{w}=0\right)$ On utilise pour cela lappareil de Richards, présenté sur la figure 2.

Il est possible d'appliquer les conditions de pression décrites précédemment en plaçant l'échantillon dans une cellule dont la base est une pierre poreuse spéciale (high air entry value), dont les pores sont de dimension suffisamment faibles pour ne pas être désaturés par les pressions d'air qu'on applique dans la cellule. L'expérimentation consiste alors à laisser l'échantillon sous pression jusqu'à ce que l'équilibre soit atteint, ce qui se produit, pour des échantillons centimétriques tels que celui de la figure 2 , généralement en quatre jours. Une fois l'équilibre atteint, on pèse l'échantillon afin de pouvoir calculer, à la fin, sa teneur en eau; on remet ensuite le même échantillon sous une pression d'air, inférieure si on s'intéresse à la réhumectation, supérieure dans le cas de la dessication.

La figure 3 (KASSIFF et al., 1969) montre ce type de courbes dans le cas d'une argile, d'un limon et d'un sable. Les valeurs de succion sont représentées en ordonnée logarithmique, soit en $\mathrm{kPa}$, soit avec la notation $\mathrm{pF}$ suivie d'un chiffre, quî est le logarithme décimal

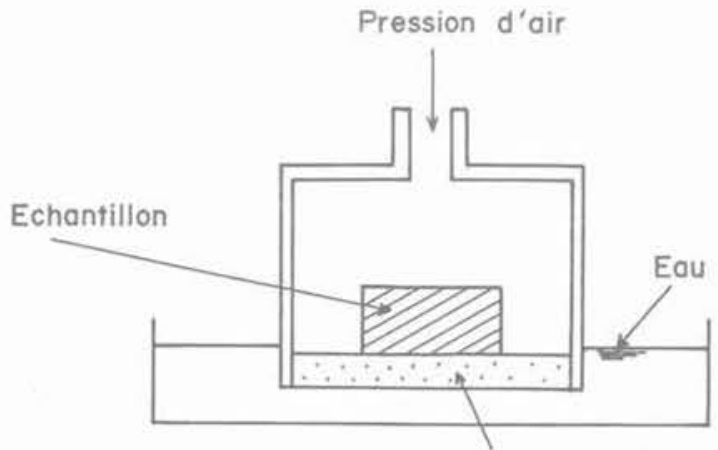

Pierre poreuse speciole

Fig. 2. - Appareil de Richards pour la détermination des courbes de rétention d'eau.

Fig. 2. - Richards apparatus for determining water retention curves.

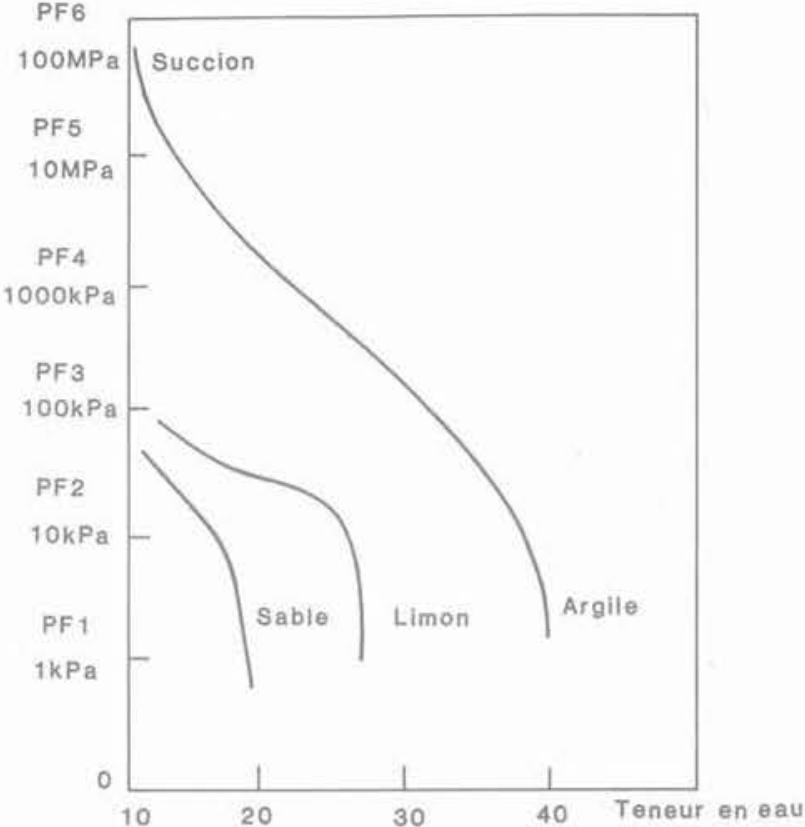

Fig. 3. - Courbes de rétention d'eau de divers types de sols (KASSIFF et al., 1979).

Fig. 3. - Water retention curves of various soils (KASSIF et al., 1979).

de la pression exprimée en $\mathrm{cm}$ d'eau. La notation $\mathrm{pF}$ est très utilisée en pédologie, où on s'intéresse aux couches de quelques dizaines de centimètres de sol au contact de la surface et qui, même dans les pays tempérés, sont soumises à des cycles de séchageremouillage qui entraînent de grandes variations de la succion et qui rendent adaptée une notation de type logarithmique.

On observe sur la figure 3 que les variations de succion sont plus importantes pour une argile que pour un limon ou un sable; pour une argile, la valeur de la succion peut atteindre des valeurs fortes d'une part du fait des interactions d'adsorption décrites plus haut, et d'autre part du fait que, les particules étant de dimensions plus faibles, il pourra se développer dans les pores qu'elles délimitent des ménisques de rayons de courbure très faibles. A titre d'exemple, sachant que l'épaisseur d'une plaquette de kaolinite qui correspondrait à l'empilement de 100 feuillets élémentaires, est égale à $0.07 \mu \mathrm{m}$, on en déduit le rayon de courbure entre deux plaquettes sera de $0,035 \mu \mathrm{m}$ et entraînera, d'après la loi de Jurin, une valeur de succion de $4285 \mathrm{kPa}$, soit pF 3,63 .

Une autre différence importante entre sols fins et sols grenus soumis aux cycles dessication-réhumectation est due aux variations de volume caractéristique des sols fins. Dans ce cas, la courbe de rétention d'eau concerne un matériau déformable dont la densité varie avec la teneur en eau. Le cas extrême est celui des sols gonflants, où une succion peut se développer sans qu'il $y$ ait désaturation. L'origine du gonflement peut dans ce cas être soit d'origine minéralogique (présence de montmorillonite), soit mécanique (argile surconsolidée). Les variations de volume se produisent, au cours du séchage, jusqu'à ce que la limite de retrait soit atteinte. 
Dans le cas des sols fins compactés, la densité initiale a une influence sur lallure de la courbe, et pour un sol donné à une teneur en eau donnée, la succion sera d'autant plus faible que la densité sera forte.

Les sols grenus, du fait de leur faible compressibilité, présenteront de faibles modifications de leur squelette solide lors des cycles qui auront lieu à densité presque constante. De plus, l'ordre de grandeur des variations de succion est beaucoup plus faible, et est influencé par la granulométrie et la densité relative.

La figure 4 (CRONEY 1952) illustre dans le cas d'un sable pollué, une caractéristique fondamentale du comportement hydraulique des milieux poreux non saturés, qui est l'hystérésis de la courbe dessicationréhumectation. Ainsi, suivant le chemin de teneur en eau parcouru par un massif de sol soumis aux conditions climatiques, on peut, pour une même teneur en eau, aboutir à des succions différentes. Dans la mesure où la succion a une influence sur le comportement mécanique, on réalise la difficulté supplémentaire engendrée par ce phénomène.

La connaissance des courbes de rétention d'eau est de première importance pour la compréhension des phénomènes hydrauliques, et donc mécaniques qui affectent un massif de sol non saturé.

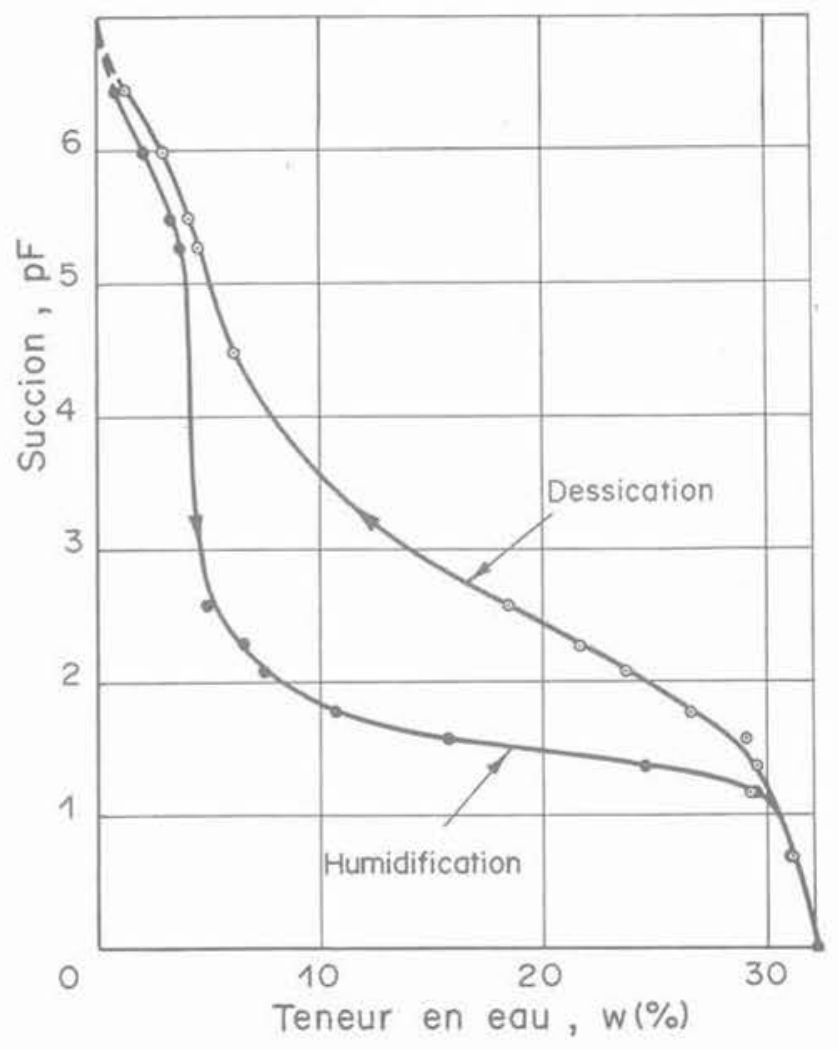

Fig. 4. - Hystérésis des courbes de rétention d'eau (CRONEY, 1952).

Fig. 4. - Hysteresis of water retention curves (CRONEY, 1952).

\section{Mesure de la succion}

On a vu que les courbes de rétention d'eau étaient obtenues non par mesure, mais par contrôle de la valeur de la succion; la mesure de cette grandeur physique est en effet délicate, et il n'existe pas actuellement de procédure simple à mettre en ceuvre et qui permette de balayer avec une précision satisfaisante toute la gamme des valeurs de succion rencontrées dans les sols.

Pour des valeurs de la succion faibles et inférieures en valeur absolue à la pression atmosphérique, on utilise la tensiométrie, qui repose sur un principe de mesure analogue à celui des pressions positives : dans un capteur électronique à membrane fine dont on mesure la déflexion par jauges de déformations, l'eau en dépression entraînera une déflexion de sens contraire à celle engendrée par les pressions positives. Dans ces conditions, l'eau naturelle, non pure et non désaérée, présente vers $70 \mathrm{kPa}(0,7$ atmosphère $)$ le phénomène de cavitation, et la mesure ne peut plus être effectuée pour des valeurs de succions supérieures. Cette gamme de mesure est donc satisfaisante pour les sables mais insuffisante pour les sols fins.

Une autre méthode de mesure, la psychrométrie (SPANNER, 1951; VERBRUGGE, 1974) est basée sur la relation de Kelvin (2) entre l'humidité relative au voisinage d'un ménisque et le rayon de ce ménisque. C'est une mesure de l'humidité relative de la phase gazeuse, au moyen d'un thermocouple utilisé de la façon suivante : dans un premier temps, la jonction du thermocouple est refroidie par effet Peltier en dessous du point de rosée, et il y a condensation d'eau liquide ; on laisse ensuite l'évaporation se produire, ce qui entraîne un abaissement de la température au niveau de la jonction, et une force électromotrice due à l'effet Seebeck, dont on calibre l'évolution à partir de conditions d'humidité relatives et de succions connues. Il s'agit d'une méthode assez sophistiquée dont les applications pratiques en mécanique des sols sont encore assez rares (VERBRUGGE, 1978; EDIL et al., 1981).

\section{HYDRAULIQUE DES SOLS NON SATURÉS}

Il est établi que la loi de Darcy s'applique aux milieux poreux non saturés indéformables, en considérant un coefficient de perméabilité variable en fonction de la teneur en eau :

$$
\underline{\mathrm{V}}=-\mathrm{k}(\theta) \underline{\operatorname{grad}} \mathrm{H}
$$

où $\theta$ est la teneur en eau volumique.

Dans le cas d'une nappe non saturée soumise uniquement à l'action de la pesanteur, le potentiel $H$ est la somme du potentiel gravitaire, et du potentiel matriciel défini par la courbe de rétention d'eau (figure 3 ). On a donc :

$$
\mathrm{H}=\mathrm{z}+\psi(\theta)
$$


En écrivant l'équation de continuité :

$$
\operatorname{div} \underline{V}=-\frac{\partial_{\theta}}{\partial t}
$$

on obtient l'équation de Richards (1931), qui gouverne les écoulements en milieux poreux non saturés, et qui est exprimée ici sous forme unidimensionnelle :

$$
\frac{\partial \theta}{\partial t}=\operatorname{div}[D(\theta) \underline{\operatorname{grad}} \theta]+\frac{\partial K(\theta)}{\partial z}
$$

avec

$$
(\theta)=K(\theta) \frac{d \psi}{\partial \theta} .
$$

En résolvant cette équation sous forme bidimensionnelle, par la méthode des différences finies, VAUCLIN et al. (1976) ont modélisé le comportement d'une nappe à surface libre soumise à des drainages et recharges, et ont pu vérifier expérimentalement sur modèle réduit la validité de leurs calculs.

\section{Mesures de perméabilité}

Les mesures de perméabilité hydrauliques de sols non saturés ont principalement été développées par les agronomes. Une méthode consiste à imposer un gradient constant dans un échantillon où une valeur connue et fixe de la succion est appliquée par contrepression d'air, et à mesurer le débit d'eau traversant l'échantillon (COREY, 1957). D'autres chercheurs ont déduit les valeurs de perméabilité des variations au cours du temps des quantités d'eau sortant de l'appareil de Richards (fig. 2) lors d'une augmentation de la pression d'air (GARDNER, 1956). Enfin a également été développée la méthode du profil instantané, qui consiste à imposer un drainage ou un remouillage à une des extrémités d'une colonne verticale de sol, en mesurant, par tensiométrie ou psychrométrie, les variations de la succion et de la teneur en eau en fonction de la hauteur et du temps. Le gradient hydraulique est déduit, en chaque point, des variations de la succion en fonction de la hauteur, et le débit est calculé d'après les variations de la teneur en eau en chaque point en fonction du temps (VACHAUD et al., 1974; DANIEL, 1982).

Les mesures de perméabilité à l'air se font en appliquant de part et d'autre d'une éprouvette de teneur en eau donnée, un gradient de pression d'air, et en mesurant les débits.

Un exemple de variations des perméabilités à l'eau et à lair en fonction du degré de saturation est représenté figure 5 , en perméabilités relatives.

On observe que, pour l'échantillon considéré, la perméabilité à l'eau est négligeable pour un degré de saturation compris entre 0 et $25 \%$, plage dans laquelle il n'y a pas de continuité du film d'eau, alors que la phase gazeuse est elle-même continue. Au-dessus de $75 \%$ de saturation, la perméabilité à l'eau devient non négligeable par rapport à la perméabilité à l'état saturé.

Sachant que de façon générale les variations de perméabilité hydraulique dans les sols ont des plages de variations de plusieurs ordres de grandeur, il est inté-

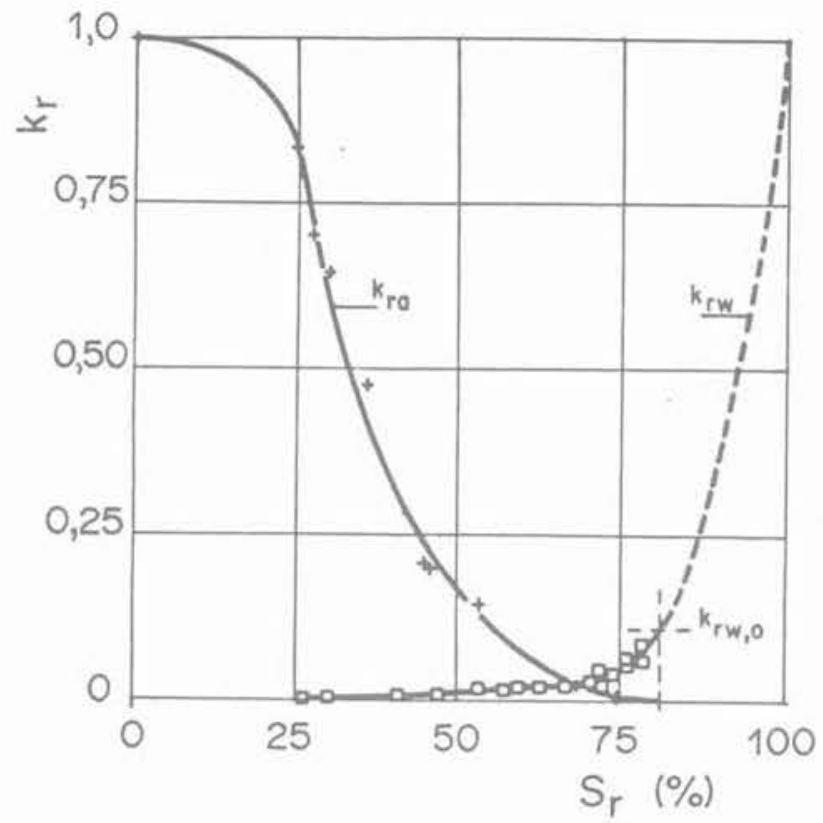

Fig. 5. - Perméabilités d'un sol non saturé
(VACHAUD et al., 1974).

Fig. 5. - Permeabilities of a non-saturated soil (VACHAUD et al., 1974).

ressant d'utiliser une ordonnée logarithmique pour les perméabilités relatives, afin de préciser davantage les phénomènes pour de faibles perméabilités. On obtient ainsi des résultats tels que ceux présentés en figure 6 (DANIEL, 1982), qui montrent qu'on a couramment dans les sols fins des variations de perméabilité de 4 à 6 ordres de grandeur, avec des courbes moyennes d'allures variables.

La perméabilité à l'air (fig. 5) décroît, dans le cas considéré, principalement entre 0 et $50 \%$ de saturation. Elle devient nulle à $77 \%$. Il y a donc continuité de la phase gazeuse en dessous de $77 \%$, et occlusion de l'air, qui est alors présent sous forme de bulles, au-dessus de $77 \%$; la continuité simultanée des deux phases intervient donc entre 25 et $77 \%$. Cette notion d'occlusion d'air est particulièrement importante dans le cas des sols compactés. GILBERT (1959) in BARDEN (1965) a établi expérimentalement, sur l'argile silteuse de Vicksburg, que la phase gazeuse était continue à $4 \%$ en dessous de l'optimum, et entièrement occluse à $3 \%$ au-dessus.

ll ne faudrait pas déduire des résultats de la figure 4 qu'un sol à de faibles degrés de saturation peut, du fait de sa faible perméabilité hydraulique relative, s'opposer à un transfert d'eau. Il va de soi qu'un sol sec soumis à un front d'humidification voit sa perméabilité hydraulique relative atteindre rapidement 1 .

\section{ASPECTS MÉCANIQUES}

\section{Contrainte effective dans les sols non saturés}

Le problème de la validité du concept de contrainte effective pour les sols non saturés est fondamental, 

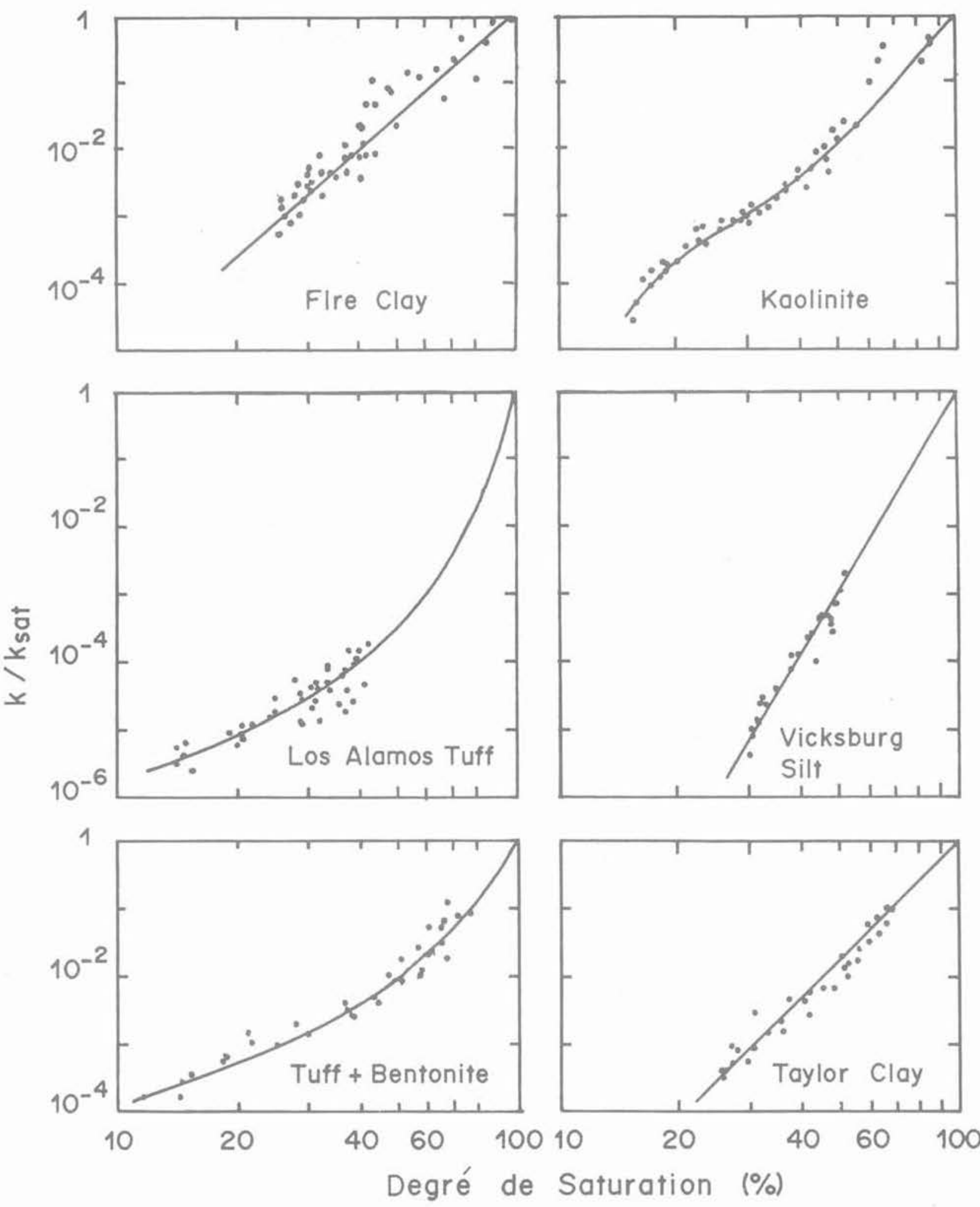

Fig. 6. - Perméabilité de divers sols non saturés (DANIEL, 1982).

Fig. 6. - Permeabilities of various non-saturated soils (DANIEL, 1982). 
puisqu'il conditionne l'utilisation des résultats déjà acquis pour les sols saturés, en particulier en ce qui concerne la modélisation du comportement du squelette du sol.

En se basant sur la définition de TERZAGHI (1936), BISHOP et BLIGHT (1963) estiment que la contrainte effective est une fonction de la contrainte totale et de la pression interstitielle qui contrôle les effets mécaniques, tels que variation de volume ou de résistance au cisaillement, dus à une modification de la contrainte appliquée à un élément de sol. Dans le cas non saturé, une telle fonction devrait également dépendre de la pression d'air $u_{a}$, et de la pression négative $u_{a}-u_{w}$.

Les difficultés rencontrées dans la généralisation du concept aux sols non saturés proviennent du fait que les pressions interstitielles, suivant qu'elles sont positives ou négatives, sont des grandeurs physiques de nature différente dont les variations n'engendrent pas, sur le squelette du sol, les mêmes sollicitations. Ainsi, une pression positive est, comme le note TERZAGHI, une contrainte * neutre "qui agit dans l'eau et dans le solide dans toutes les directions avec une intensité égale, alors qu'une pression négative ne s'applique que sur la surface mouillée du solide, qui diminue avec la désaturation, dans des directions qui dépendent de la géométrie des pores inter-granulaires; de plus, dans le cas des sols fins, les variations de pression négative engendrent une variation de l'indice des vides et de l'arrangement des particules du sol.

Une profonde réflexion a été menée par nombre de chercheurs aux alentours des années soixante. En analysant conceptuellement les phénomènes au niveau des contacts inter-grains, et en tentant d'estimer les contraintes locales engendrées par les ménisques correspondant à une succion donnée, ils ont proposé des relations reliant les valeurs de la contrainte totale appliquée au sol, de la succion, et d'une contrainte effective hypothétique dont l'expression la plus admise, celle de BISHOP (1959) est la suivante :

$$
\sigma^{\prime}=\sigma-u_{a}+\chi\left(u_{\mathrm{a}}-u_{w}\right)
$$

$\chi$ est un paramètre adimensionnel comprit entre 0 et 1 . nul dans le cas des sols secs, et égal à 1 dans le cas des sol saturés. De façon intuitive, la grandeur $\chi\left(\mathrm{u}_{\mathrm{a}}-\mathrm{u}_{\mathrm{w}}\right)$ positive, représenterait le supplément de contrainte intergranulaire d'attraction qu'un ménisque engendre entre deux grains.

Cette formulation hypothétique permet, une fois admise, de calculer le coefficient $\chi$. Des expérimentations ont été menées dans ce but, en cisaillement triaxial, par BISHOP et DONALD (1961), qui ont comparé, à la rupture, les résultats d'essais non saturés à succion $\left(u_{a}-u_{w}\right)$ constante, à ceux d'essais saturés, représentatifs du comportement effectif du squelette. Ils obtiennent ainsi des valeurs de $\chi$ en fonction du degré de saturation.

En suivant la même démarche pour des essais de compression à l'œdomètre, JENNINGS et BURLAND (1962) mettent en évidence des limitations à l'usage du concept de contrainte effective, en remarquant en particulier qu'il n'y a pas de relation unique entre la contrainte effective de BISHOP et l'indice des vides, quand on se trouve au-dessous d'un degré de saturation critique qui peut atteindre $90 \%$ pour les argiles.
Ils insistent sur le fait que toutes les méthodes utilisées pour le calcul de $X$ sont basées sur l'acceptation a priori de la formule (7). Ces valeurs, exprimées en fonction du degré de saturation, sont représentées en figure 7. On observe que l'allure des courbes est très variable, et qu'en particulier la relation $\chi=S r$ n'est pas vérifiée. JENNINGS et BURLAND explicitent ces problèmes en termes de structure, en considérant que les modifications de l'arrangement des particules dues au retrait des ménisques en cours de désaturation sont très différentes de celles créées par des contraintes extérieures. Une autre limitation à l'utilisation de $x$ est sa dépendance à l'égard du chemin de contrainte suivi lors d'un essai de cisaillement ou de compression. D'autre part. pour un sol donné, les valeurs de $\chi$ obtenues en cisaillement et en compression ne sont pas toujours identiques.

En réponse à JENNINGS et BURLAND (1962), BISHOP et BLIGHT (1963) réexaminent le concept et la relation (7), en convenant en particulier que des variations de $\mathrm{u}_{\mathrm{a}}-\mathrm{u}_{\mathrm{w}}$ sont presque toujours accompagnées de variations importantes de $\chi$; ils estiment de plus que les modifications de structure engendrées par les variations de succion ont sans doute une grande importance vis-à-vis des problèmes posés par l'utilisation du concept pour la description des variations de volume des sols non saturés, en conditions cedométriques ou isotropes. Ils suggèrent finalement une relation plus générale sous la forme:

$$
\sigma^{\prime}=\sigma-u_{a}+f\left(u_{a}-u_{w}\right)
$$

Ils rejoignent ainsi COLEMAN (1962), en proposant de représenter les propriétés de changement de volume en considérant l'indice des vides e comme une fonction des variables indépendantes $\left(\sigma-u_{0}\right)$ et $\left(u_{0}-u_{w}\right)$; il s'agit alors de savoir s'il est possible de définir une surface caractéristique des variations de

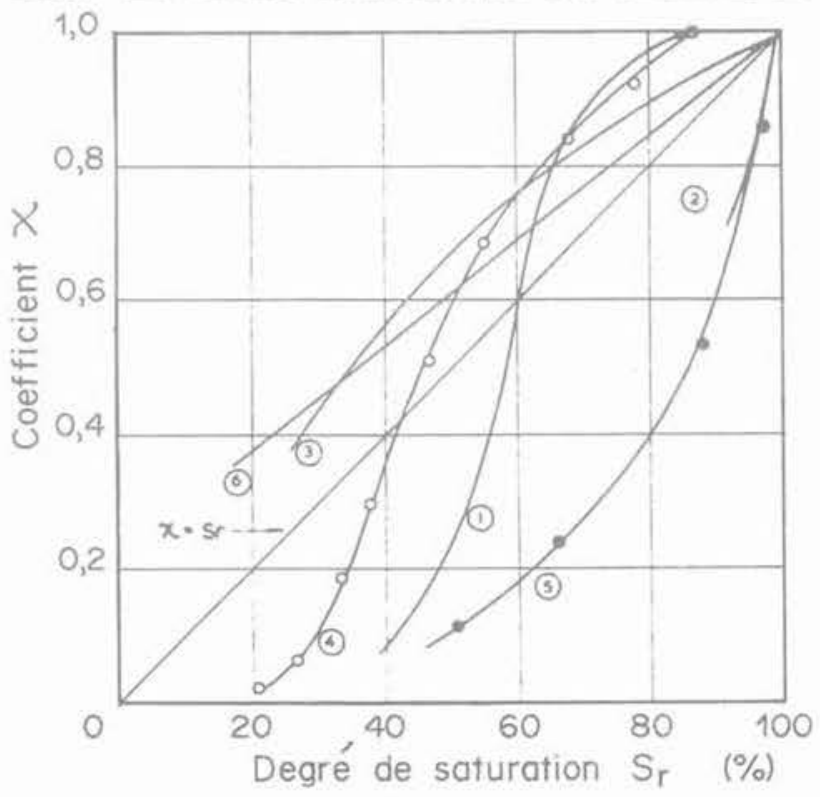

Fig. 7. - Variations de $x$ en fonction du degré de saturation (JENNINGS et BURLAND, 1962).

Fig. 7. - Variations of the $x$ coefficient as a function of the saturation degree

(JENNINGS and BURLAND, 1962). 


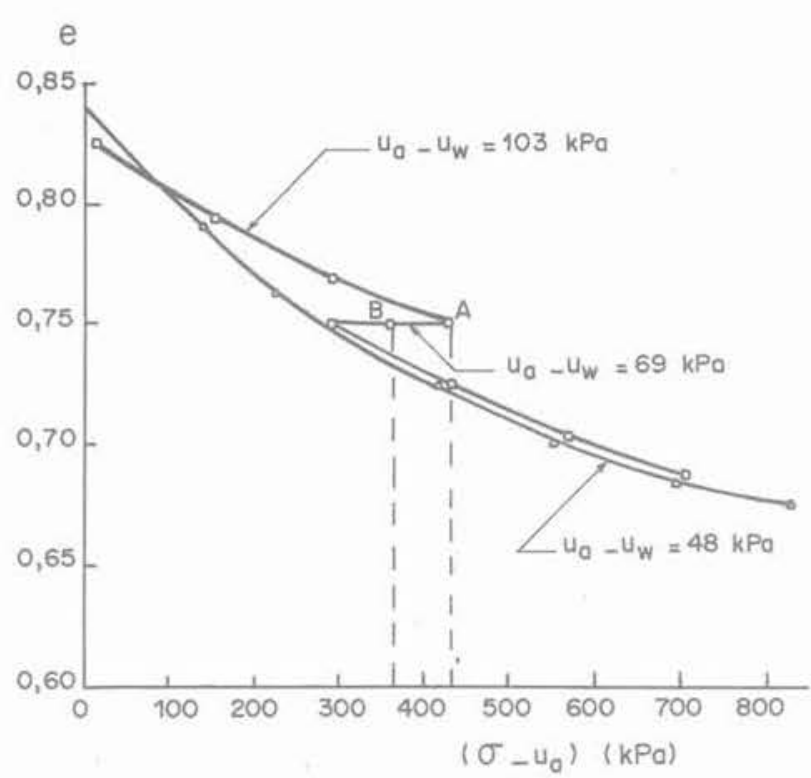

Fig. 8. - Exemple de calcul de $x$ (MATYAS et RADHAKRISHNA, 1968).

Fig. 8. - Calculation of $x$ in a particular case (MATYAS and RADHAKRISHNA, 1968).

l'indice des vides dans cet espace, moyennant certaines restrictions sur le chemin de contrainte et lhistoire de la saturation. En termes de résistance au cisaillement, ils considèrent que le concept est plus adapté, et proposent des relations $\chi / \mathrm{Sr}$ établies expérimentalement.

Une mise en évidence particulièrement explicite des limitations à l'usage de $\chi$ a été proposée par MATYAS et RADHAKRISHNA (1968), et est présentée figure 8. On y observe, en compression isotrope, deux courbes de variation de l'indice des vides à succion constante en fonction de la contrainte appliquée $\sigma-u_{a}$. Un chemin (1) est continu sous une succion de $48 \mathrm{kPa}$, pour des contraintes allant de 0 à $800 \mathrm{kPa}$. Le chemin 2 correspond à une succion de $103 \mathrm{kPa}$ jusqu'à un indice des vides de 0,75 , où la succion passe à 69 , puis $48 \mathrm{kPa}$, et les deux courbes coincident. Entre les points $A$ et $B$, le chargement de succion se fait sans variation de volume. D'après le principe de contrainte effective, on aurait :

$$
\mathrm{d} e=\mathrm{C} d \sigma^{\prime}=0
$$

La relation de Bishop peut s'écrire :

$$
\Delta \sigma^{\prime}=\Delta\left(\sigma_{3}-\mathrm{u}_{\mathrm{a}}\right)+\chi \Delta\left(\mathrm{u}_{\mathrm{a}}-\mathrm{u}_{\mathrm{w}}\right)=0
$$

d'où

$$
X=-\frac{\Delta\left(\sigma_{3}-u_{a}\right)}{\Delta\left(u_{a}-u_{w}\right)}=-\frac{427-359}{103-69}=-2
$$

ce qui est contraire à la définition, où $0<\chi<1$. L'ensemble de ces considérations contribuent à engendrer un certain doute sur la validité du concept de contrainte effective et du coefficient X. Il est donc nécessaire d'adopter une certaine prudence dans l'utilisation de ce concept pour se ramener à de pseudo- caractéristiques de squelette sur lesquelles on appliquerait des modélisations de comportement, classiques en saturé.

Ce doute a conduit nombre d'auteurs à adopter rapidement, comme le suggère COLEMAN (1962), des approches en variables indépendantes (BISHOP et BLIGHT, 1963; MATYAS et RADHAKRISHNA, 1968; FREDLUND et MORGENSTERN, 1977), en particulier en ce qui concerne la compressibilité et la consolidation des sols compactés ou non saturés.

\section{Consolidation}

Un certain nombre de travaux expérimentaux ont été menés sur la compressibilité des sols compactés (JENNINGS et BURLAND, 1962; YOSHIMI et OSTERBERG, 1963 ; MATYAS et RADHAKRISHNA, 1968; BARDEN et al., 1969). La grande complexité du comportement du milieu triphasique a été mise en évidence, avec plus particulièrement les problèmes dus à l'hystérésis séchage-remouillage, et à ses conséquences sur la structure des sols fins. La figure 9 (MATYAS

(4)

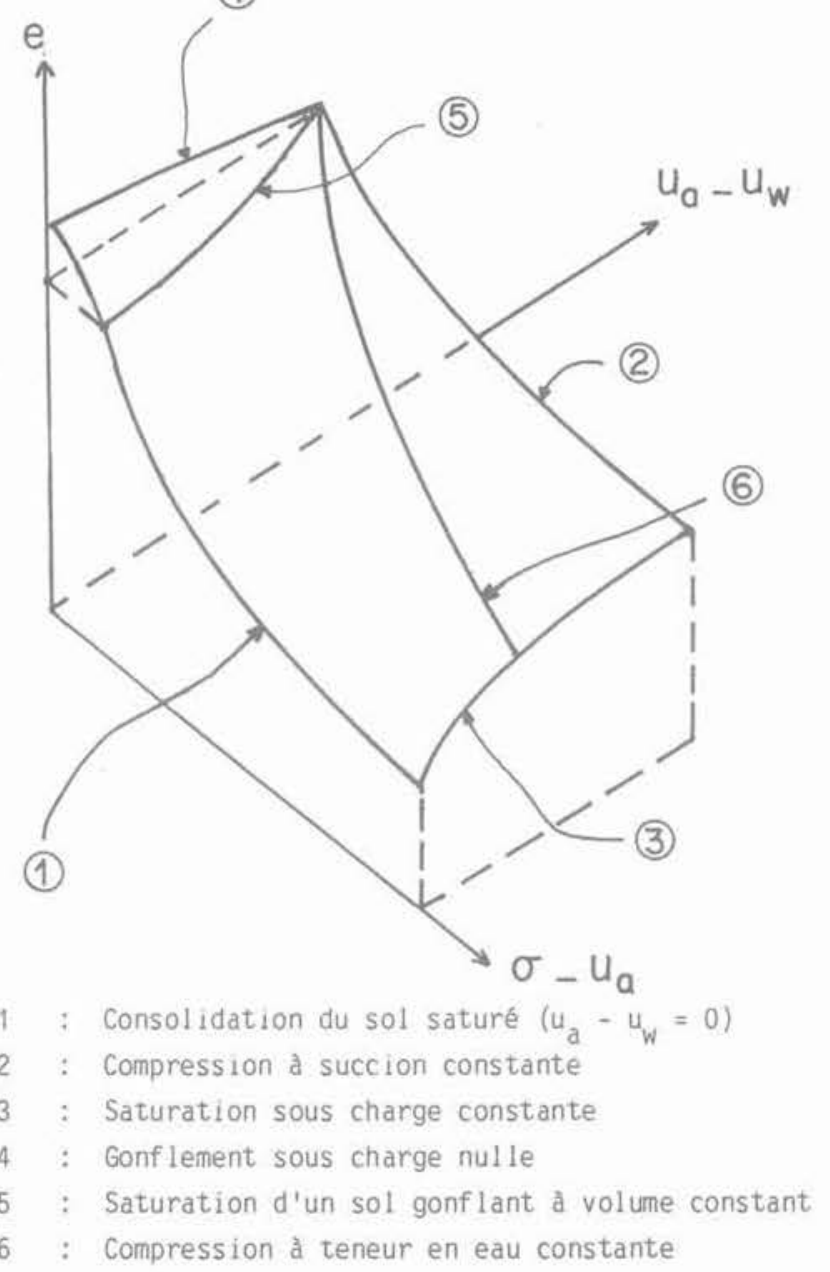

Fig. 9. - Utilisation

de deux variables indépendantes (MATYAS et RADHAKRISHNA, 1968).

Fig. 9. - Use of two independent variables (MATYAS and RADHAKRISHNA, 1968). 
et RADHAKRISHNA, 1968) est basée sur l'approche en variables indépendantes, et fournit une bonne synthèse de l'influence des variations de contraintes totales et de succion sur l'indice des vides d'un sol non saturé. L'existence d'une telle surface est conditionnée par la nécessité de chemins à variations monotones du degré de saturation. L'allure de cette surface montre, en comparant le chemin de consolidation du sol saturé 1 . à celui à succion non nulle constante 2 , dont la pente e/ $\left(\sigma-u_{a}\right)$ est moins forte, que la compressibilité diminue quand la valeur de la succion augmente, c'est-à-dire quand le sol est plus sec; le chemin 3 à contrainte constante avec diminution de la succion correspond à un remouillage sous charge constante, et la figure présente un cas d'effondrement; le chemin 4 est caractéristique d'un remouillage sous contrainte nulle, qui se produit avec augmentation de l'indice des vides, et représente un gonflement. Le chemin 5 est obtenu par saturation à volume constant, et son intersection avec le plan $\left(\pi-u_{a}, e\right)$ donne la valeur de la contrainte de gonflement dans ces conditions. Un essai de compressibilité classique de sol compacté (6) suffisamment sec se fait pour une valeur de la succion $\mathrm{u}_{\mathrm{a}}-\mathrm{u}_{w}$ qui reste positive, sans expulsion d'eau, à teneur en eau constante, avec augmentation du degré de saturation et diminution de la succion. MATYAS et RADHAKRISHNA établissent également l'existence d'une surface $\mathrm{Sr}=\mathrm{g}\left(\sigma-\mathrm{u}_{\mathrm{a}}, \mathrm{u}_{\mathrm{a}}-\mathrm{u}_{\mathrm{w}}\right)$.

Malgré sa restriction en termes de variation de degré de saturation, on constate que cette notion de surface d'état caractérisant les variations de volume ou de degré de saturation d'un sol non saturé en fonction des variables indépendantes $\left(\sigma-u_{a}\right)$ et $\left(u_{a}-u_{w}\right)$ semble être pleine de potentialités. Il semble à présent souhaitable de déterminer ces surfaces pour divers échantillons, pour confirmer et généraliser ce concept.

\section{Approches théoriques}

Diverses approches théoriques de la consolidation des sols non saturés ont été développées. Celle de BARDEN (1965) présente l'intérêt de reposer sur une analyse très détaillée des phénomènes physiques intervenant et des lois les gouvernant, dans les différentes plages de degrés de saturation. Les lois physiques adoptées sont la loi de Darcy, pour les transferts d'eau et d'air, et la loi de Henry. pour la dissolution de l'air dans l'eau. L'équation de continuité appliquée à l'air et à l'eau donne deux équations. Sur le plan mécanique, et tenant compte des restrictions apportées par BISHOP et BLIGHT (1963) au concept de contrainte effective, BARDEN considère que la porosité n est une fonction inconnue de la contrainte, du degré de saturation $\mathrm{Sr}$ et d'un paramètre $\lambda$, représentatif de la structure. La complexité de cette relation le conduit à proposer des solutions simplifiées pour les diverses plages de degré de saturation qu'il considère, et qui sont les suivantes :

(a) argile très sèche $\left(\mathrm{Sr}_{r}<50 \%\right)$ : l'eau est solidement liée aux grains solides, et l'air est le seul fluide en mouvement lors de la compression;

(b) argile du côté sec de loptimum $(50 \%<\mathrm{Sr}<90 \%)$ : la succion $\mathrm{u}_{\mathrm{a}}-\mathrm{u}_{\mathrm{w}}$ est encore assez forte pour qu'elle n'atteigne par zéro en cours de compression, il y a continuité de l'air, et l'eau n'est pas expulsée; (c) autour de l'optimum : il y a "probablement » une phase transitoire avec transfert simultané d'air et d'eau, apparition d'une pression d'eau positive et expulsion d'eau; l'augmentation de Sr fait chuter la perméabilité à l'air et augmenter la perméabilité à l'eau;

(d) du côté humide de l'optimum ( $\mathrm{Sr}>90 \%$ ) : l'air est occlus et ne peut pas s'écouler séparément, ce qui rend très faible la perméabilité à l'air; une part de l'air est piégée dans le squelette, l'autre se mélange à l'eau sous forme de petites bulles, et engendre un fluide compressible:

(e) argile très humide $(\mathrm{Sr}>95 \%$ ) : le peu d'air présent est piégé par le squelette.

Les simplifications adoptées dans chacune de ces plages de saturation reviennent cependant le plus souvent à adopter la relation de Bishop. Le système d'équations obtenu est alors résolu numériquement, et les solutions trouvées sont comparées à la solution de Terzaghi pour les sols saturés.

FREDLUND et HASAN (1979) proposent une approche en variables indépendantes $\left(\mathrm{u}_{\mathrm{a}}-\mathrm{u}_{\mathrm{w}}\right)$ et $\left(\sigma-\mathrm{u}_{\mathrm{a}}\right)$, moins proche de la physique des phénomènes que celle de BARDEN (1965), avec en particulier l'hypothèse de la continuité de la phase gazeuse qui, d'après l'étude de BARDEN, ne devrait correspondre qu'aux argiles compactées du côté sec de l'optimum $(\mathrm{Sr}<90 \%)$. La plage de degrés de saturation où cette hypothèse est valable peut d'ailleurs être identifiée par la détermination des variations de perméabilités à l'air et à l'eau. Dans le cas de la figure 5, il n'y a continuité des deux phases qu'entre 25 et $80 \%$ de saturation. FREDLUND et HASAN (1979) font en outre l'hypothèse de la constance des coefficients de perméabilité à l'air et à l'eau pendant la consolidation. et négligent, comme BARDEN, la diffusion de l'air dans l'eau.

Ils écrivent l'équation de continuité de l'eau et de l'air, et font l'hypothèse de l'existence d'une surface caractéristique des variations de volume total $\frac{\Delta V}{V}$, et du volume d'eau $\frac{\Delta V_{w}}{V}$, dans des espaces où les deux autres variables sont $\sigma-\mathrm{u}_{\mathrm{a}}$ et $\mathrm{u}_{\mathrm{a}}-\mathrm{u}_{\mathrm{w}}$. Ils débouchent ainsi sur des équations de diffusion pour l'air et pour l'eau qu'ils résolvent numériquement en proposant, comme BARDEN, des courbes adimensionnelles qui sont comparées à la solution de Terzaghi.

LLORET et ALONSO (1980) développent également une solution en variables indépendantes, basée sur une étude bibliographique détaillée, et utilisant les résultats expérimentaux de MATYAS et RADHAKRISHNA (1968), qui définissent des surfaces caractéristiques de la porosité $n$ et du degré de saturation $\mathrm{Sr}$ en fonction de $\left(\sigma-u_{a}\right)$ et $\left(u_{a}-u_{w}\right)$, pour des variations monotones du degré de saturation. Ils intégrent d'autre part les différents états de l'air, suivant qu'il est continu ou occlus, en tenant compte des variations de perméabilité à l'air et à l'eau en fonction du degré de saturation : ils observent en particulier que cette perméabilité à l'air devient très faible dans le cas considéré, pour des valeurs de $\mathrm{Sr}$ supérieures à $85 \%$, ce qui est en bonne concordance avec les travaux de BARDEN (1965), et la figure 5 . 
Les équations sont obtenues à partir des équations de continuité de l'air et de l'eau, de l'application de la loi de Darcy pour ces deux fluides, des courbes expérimentales de variation de perméabilité à l'eau et à l'air en fonction du degré de saturation, et des surfaces déterminées par MATYAS et RADHAKRISHNA (1968). La méthode numérique de résolution est décrite, et le modèle proposé est ensuite appliqué à différents cas de consolidation, infiltration, gonflement, et effondrement.

Il reste cependant à confronter les approches théoriques aux résultats expérimentaux, afin de les valider pour pouvoir ensuite envisager leur application pour décrire le comportement d'ouvrages en sols compactés tels que remblais ou barrages en terre.

\section{Résistance au cisaillement}

C'est à partir d'essais de cisaillement à l'appareil triaxial que le coefficient $\chi$ de Bishop a été introduit. Ces essais (BISHOP et DONALD, 1961) ont été réalisés sur un appareillage spécial permettant l'établissement d'une succion connue et constante au moyen d'une surpression d'air, la pression de l'eau restant égale à la pression atmosphérique; pour cela, le principe de l'appareil de Richards (fig. 2) était adapté sur une cellule triaxiale qui utilisait de plus du mercure comme fluide de remplissage, pour éviter des pertes d'air au travers de la membrane entourant l'échantillon.

Les données expérimentales sur l'influence de la succion sur la résistance au cisaillement sont assez rares. VERBRUGGE (1978) a testé la méthode psychrométrique pour mesurer la succion au cours d'essais de cisaillement triaxiaux, et mis en évidence une augmentation du module et une diminution de la déformation à la rupture dus à l'augmentation de la succion. Il analyse ses résultats en termes de contraintes effectives, en déterminant les variations de $\chi$ en fonction de Sr. ESCARIO (1980) présente une boîte de cisaillement directe à succion contrôlée par surpression d'air, et ESCARIO et SAEZ (1986) présentent des résultats sur deux argiles, dont une gonflante, et un sable argileux. Ils observent sur les argiles une tendance à l'augmentation de l'angle de frottement apparent avec la succion, dans un diagramme $\tau, \sigma-u_{0}$. Pour le sable argileux, cet angle ne semble pas varier avec la succion.

FREDLUND et al. (1978) exploitent des résultats d'essai déjà publiés, en variables indépendantes. En faisant l'hypothèse que, dans un espace à trois dimensions, $\tau \sigma-u_{w}$ et $u_{a}-u_{w}$, les points caractéristiques de la rupture sont situés sur une surface plane, ils définissent un critère de rupture qui prend la forme suivante :

$$
\tau=c^{\prime}+\operatorname{tg} \varphi^{\prime}\left(\sigma-u_{a}\right)+\operatorname{tg} \varphi^{b}\left(u_{a}-u_{w}\right)
$$

ils introduisent ainsi deux angles de frottement, correspondant à l'intersection de la surface de rupture supposée plane et des plans $\left(\tau, \sigma-u_{a}\right)$ et $\left(\tau, u_{a}-u_{w}\right)$.

Le caractère plan de cette surface ne semble cependant pas établi expérimentalement, et des premiers résultats de VERBRUGGE (1979) semble montrer qu'il n'y a pas unicité de l'angle $\varphi^{b}$ défini par FREDLUND et al. (1978). La même remarque est déduite de l'observa- tion des résultats de ESCARIO et SAEZ (1986), qui mettent en évidence une variation de langle de frottement apparent avec la succion, qui est contraire à lhypothèse de surface plane de FREDLUND et al.

Une autre conséquence découle de cette hypothèse, obtenue en observant que la relation 12 peut également s'écrire sous la forme:

$$
\tau=c^{\prime}+\operatorname{tg} \varphi^{\prime}\left[\left(\sigma-u_{a}\right)+\frac{\operatorname{tg} \varphi^{b}}{\operatorname{tg} \varphi^{\prime}}\left(u_{n}-u_{w}\right)\right]
$$

en posant $\chi=\frac{\operatorname{tg} \varphi^{b}}{\operatorname{tg} \varphi^{\prime}}$, on retombe sur lexpression de la contrainte effective de Bishop.

Il reste donc un travail expérimental important à effectuer pour comprendre l'influence de la succion sur la rêsistance au cisaillement des sols.

\section{CONCLUSION}

Ce travail présente un certain nombre de concepts nécessaires pour aborder le comportement des sols non saturés. Sur un plan hydraulique, les phénomènes affectant les milieux poreux non saturés incompressibles ont fait l'objet de nombreux travaux dans des disciplines connexes, et un certain nombre de résultats importants ont été établis. Les sols sont caractérisés par leur déformabilité, et le couplage mécaniquehydraulique pose de nombreux problèmes, dont l'ampleur est augmentée par l'hystérésis des cycles de dessication-réhumectation.

Le concept de contrainte effective a fait l'objet de travaux approfondis au début des années soixante, qui ont conduit la plupart des auteurs, au moins en ce qui concerne l'étude des variations de volume, à adopter des approches où la succion est considérée comme une variable indépendante. Dans des conditions de variations monotones du degré de saturation, le concept de surface d'état définissant la porosité et le degré de saturation en fonction de la contrainte appliquée et de la succion semble prometteur. Les modélisations existantes des phénomènes de consolidation des sols compactés sont très sophistiquées et il est probable que l'apport expérimental permettra des simplifications justifiées.

Dans le domaine de la résistance au cisaillement, les quelques approches proposées reposent sur de faibles bases expérimentales, et le travail à accomplir reste considérable.

Il reste que le besoin d'une meilleure connaissance du comportement des sols non saturés se fait actuellement ressentir, en particulier pour parvenir à la compréhension de troubles affectant certains ouvrages en terre tels que remblais ou barrages, conçus sans tenir compte de la non-saturation; cette meilleure connaissance devrait également permettre à l'avenir un meilleur dimensionnement de ces ouvrages.

\section{REMERCIEMENTS}

Cette étude a été menée en collaboration avec l'Université de Haifa (Israël), grâce au soutien de la DAEI (MELATT), et de la Société Scetauroute. 


\section{BIBLIOGRAPHIE}

1. BARDEN L. (1965), Consolidation of compacted and unsaturated clays, Géotechnique, 15 (3), pp. $267-286$

2. BISHOP A.W. (1959), Teknisk Ukeblad, $n^{\circ} 39$, pp. 859-863.

3. BISHOP A.W. and BLIGHT G.E. (1963), Some aspects of effective stress in saturated and unsaturated soils, Géotechnique n 3, pp. 177-197.

4. BISHOP A.W. and DONALD I.B. (1961), The experimental study of partly saturated soil in the triaxial apparatus, Proc. 5th I.C.S.M.F.E. 1, pp. 13-21, Paris.

5. COLEMAN J.D. (1962), Stress strain relations for partly saturated soil. Correspondence, Géotechnique $12, n^{\circ} 4$, pp. 348-350.

6. COREY E.C. (1957), Measurement of air and water permeability in unsaturated soil, Proceeding of the Soil Science Society of America, 21. pp. 7-11.

7. CRONEY D. (1952), The movement and distribution of water in soils, Géotechnique, 3 (1) pp. 1-16.

8. DANIEL D.E. (1982), Measurement of hydraulic conductivity of unsaturated soils with thermocou. ple psychrometers, Soil Science Society of America Journal, $20 n^{\circ} 6$ pp. 1125-1129.

9. EDIL T.B., MOTAN S.E. and TOHA F.X. (1981), Mechanical behaviour and testing methods of unsaturated soils, Laboratory Shear Strength of Soil, A.S.T.M., S.T.P. 740, pp. 114-129.

10. ESCARIO V. (1980), Suction controlled penetra. tion and shear tests. Proceedings of the 4th International Conference on Expansive Soils II, pp. 781-797, Denver, A.S.C.E.

11. ESCARIO V. and SAEZ J. (1986), The shear strength of partly saturated soils, Géotechnique 36 (3), pp. 453-456.

12. FREDLUND D.G. and MORGENSTERN N.R. (1977). Stress state variables for unsaturated soils. Journal of Soil Mechanics and Foundation Engineering, A.S.C.E., G.T.5, pp. 447-466.

13. FREDLUND D.G., MORGENSTERN N.R. and WIDGER A. (1978), Shear strength of unsatura. ted soils, Canadian Geotechnical Journal, vol. 15, $n^{\circ} 3,1978$, pp. 313-321.

14. FREDLUND D.G. and HASAN J.V. (1979), One dimensional consolidation theory: unsaturated soils, Canadian Geotechnical Journal, 16, pp. 521-531.
15. GARDNER R. (1956), Calculation of capillary conductivity from pressure plate outflow data, Proceedings of the Soil Science Society of America, 20, pp. 317-320.

16. JENNINGS J.E.B. and BURLAND J.B. (1962), Limitations to the use of effective stresses in partly saturated soils, Géotechnique 12, $\mathrm{n}^{\circ} 2$. pp. 125-144.

17. KASSIF G.. LIVNEH $M$. et WISEMAN G. (1969), Pavements on expansive clays, Jerusalem Academic Press, $218 \mathrm{p}$.

18. LLORET A. and ALONSO E.E. (1980), Consolidation of unsaturated soils including swelling and collapse behaviour, Géotechnique $30, \mathrm{n}^{\circ} 4$, pp. $449-477$

19. MATYAS E.L. and RADHAKRISHNA H.S. (1968), Volume change characteristics of partially saturated soils, Géotechnique 18. $\mathrm{n}^{\circ} 4$. pp. $432-448$.

20. MITCHELL J.K. (1976), Fundamentals of Soil Behaviour, J. Wiley. New York, 422 p.

21. SPANNER D.C. (1951). Journal of Experimental Botany, vol. 2, n 5. pp. 145-168,

22. TERZAGHI K. (1936), The shearing resistance of saturated soils and the angles between the planes of shear, Proceedings of the First International Conference on Soil Mechanics 1, pp. 54-56.

23. TOUMA J. and VAUCLIN M. (1986), Experimental and numerical analysis of two-phase infiltration in a partially saturated soil, Transport in Porous Media 1, pp. 27-55.

24. VAUCLIN M., KHANJI D., et VACHAUD G. (1976), Etude expérimentale et numérique du drainage et de la recharge des nappes à surface libre, avec prise en compte de la zone non satu. rée, Journal de Mécanique, vol. $15, \mathrm{n}^{\circ} 2$. pp. 307-348.

25. VERBRUGGE J.C. (1974), Contribution à la mesure de la succion et de la pression interstitielle dans les sols non saturés. Thèse de doctorat, Université libre de Bruxelles, $212 \mathrm{p}$.

26. VERBRUGGE J.C. (1978), Emploi d'une méthode psychrométrique dans des essais triaxiaux sur un limon remanié non saturé. Revue Canadienne de Géotechnique, vol. 15, pp. 501509.

27. VERBRUGGE J.C. (1979), Emploi d'une méthode psychrométrique dans des essais triaxiaux sur un limon remanié non saturé: réponse, Revue Canadienne de Géotechnique vol. 16, pp. 615-616.

28. YOSHIMI Y. and OSTERBERG J.O. (1963), Compression of partially saturated cohesive soils, Journal of the Soil Mechanics and Fondation Engineering Division, A.S.C.E. 89, S.M.4, pp. 24. 
